\title{
Multichannel Surface EMG for the Non-Invasive Assessment of the Anal Sphincter Muscle
}

\author{
R. Merlettia A. Bottin ${ }^{a} \quad$ C. Cescon ${ }^{a}$ D. Farina ${ }^{a} \quad$ M. Gazzoni ${ }^{a}$ S. Martina ${ }^{a}$ \\ L. Mesin ${ }^{a}$ M. Pozzo ${ }^{a} \quad$ A. Rainoldia,b P. Enck ${ }^{c}$ \\ a Laboratory for Engineering of the Neuromuscular System (LISiN), Department of Electronics, Politecnico di Torino, \\ Torino, and ${ }^{\mathrm{b}}$ Dipartimento di Scienze Motorie, Università di Tor Vergata e Fondazione Don Gnocchi, Roma, Italy; \\ cUniversity Hospital Tübingen, Department of General Surgery, Tübingen, Germany
}

\section{Key Words}

Surface EMG · Muscle fiber conduction velocity ·

Motor unit · External anal sphincter · Innervation zone

\begin{abstract}
Background/Aims: This work focuses on recording, processing and interpretation of multichannel surface EMG detected from the external anal sphincter muscle. The aim is to describe the information that can be extracted from signals recorded with such a technique. Methods: The recording of many signals from different locations on a muscle allows the extraction of additional information on muscle physiology and anatomy with respect to that obtained by classic bipolar recordings. Multichannel EMG methods have been recently developed for the assessment of the external anal sphincter. An anal probe was used in this study to record signals at different depths within the anal canal during contractions at different effort levels. The plug is $150 \mathrm{~mm}$ in length and $14 \mathrm{~mm}$ in diameter, holding a circumferential array of 16 equally spaced silver bar electrodes, located at a distance of $20 \mathrm{~mm}$ from the probe tip and aligned with the probe
\end{abstract}

axis. Results: Information about localization of the innervation zone, fiber length, EMG amplitude, muscle fiber conduction velocity and single motor unit analysis can be obtained from the signals recorded with the circumferential array by means of innovative signal processing techniques. Conclusions: The type of information extracted from multichannel surface EMG signals cannot be obtained with other currently available techniques. The technological innovation described in this work is promising for a further insight into the investigation of pelvic floor pathologies and rehabilitation treatments.

Copyright $@ 2004$ S. Karger AG, Basel

\section{Introduction}

Although the detection of surface EMG signals is relatively easy, the interpretation of the signal features for understanding physiological mechanisms and monitoring pathological conditions is a complex task [1]. Surface EMG signals are indeed affected by many factors whose effect on the variables extracted from the signal is often not intuitive. As an example, it has been only recently rec-

\begin{tabular}{ll}
\hline KARGER & ( ) 2004 S. Karger AG, Basel \\
0012-2823/04/0692-0112\$21.00/0 \\
$\begin{array}{l}\text { Fax +4161306 12 34 } \\
\begin{array}{l}\text { E-Mail karger@karger.ch } \\
\text { www.karger.com }\end{array}\end{array}$ & $\begin{array}{l}\text { Accessible online at: } \\
\text { www.karger.com/dig }\end{array}$
\end{tabular}

Roberto Merletti, PhD

Dip. di Elettronica, Politecnico di Torino

Corso Duca degli Abruzzi 24

IT-10129 Torino (Italy)

Tel. +39 011 4330476, Fax +39011 4330404, E-Mail roberto.merletti@polito.it 
ognized that EMG signals detected at different locations over the same muscle may have significantly different amplitudes [2, 3], which implies that electrode location is of primary importance for comparing results [4]. The difficulties in interpreting results has led, in some cases, to rather strong critiques to this non-invasive technique $[5,6]$.

The most used montage for surface EMG signal detection is the bipolar configuration, which consists in recording the difference between signals detected by two electrodes placed over the same muscle at a certain distance from each other. This detection modality has been used in many studies on the assessment of the external anal sphincter (EAS) functions [7-10], with a variety of electrode shapes, sizes, and locations. Signals recorded by the bipolar configuration are affected by anatomical, geometrical, physical and detection system parameters [11]. Among these factors, the most relevant are the thickness of the layers interposed between the electrodes and the muscle, the tissue in-homogeneities, the length of the fibers, the inter-electrode distance, the shape and size of the electrodes and the relative location and orientation of the electrodes with respect to the muscle fibers. The relevance of these factors for the interpretation of results depends on the specific muscle architecture.

Many methods are described in the literature for recording EMG signals from the EAS in bipolar configuration [9]. Comparison between recordings obtained by different systems has also been reported [7-9], with emphasis on the relevant effect that the type of recording has on the signal features [7]. The sensitivity of the recording to these factors limits the applicability of the method. In particular, it hinders the possibility of comparing results from studies that adopted different recording methods and it reduces the repeatability of the test. These limitations should be recognized when presenting clinical applications.

In recent years, there have been many efforts to overcome the limitations of the classic bipolar EMG recording technique [12]. The multichannel approach has been based on increasing the number of electrodes placed over the muscle in order to obtain a map of the potential distribution over the skin rather than a single local observation. The use of multichannel surface EMG allows to concomitantly detect bipolar EMG derivations from a number of locations over the muscle. The availability of more than one detection point may be useful for the selection of the optimal locations where to reliably extract the descriptive variables of the signal. Moreover, it provides an insight into the mechanisms of generation of the signals, which may help in understanding and reducing the sources of artefact in the detection.

In the research field, multichannel surface EMG is being recorded from muscles of rather simple architecture. In the case of the EAS, the placement of many detection systems over the muscle presents important technological limitations. Recently, these limitations have been overcome and systems for surface EMG detection from this muscle with up to 48 electrodes have been presented $[13,14]$.

The aim of this work is to provide an overview of recent advances in surface EMG signal detection and interpretation in sphincter muscles. The paper will present the concepts behind recently developed multichannel EMG detection probes as well as the possible information that can be extracted from the signals acquired with such probes.

\section{Methods}

\section{Detection}

Muscle Anatomy and Concepts behind the Array Detection

Muscles are composed of nearly parallel fibers that constitute the contractile structural units. A motoneuron innervates a group of muscle fibers which thus constitutes the smallest functional unit of the muscle. The motoneuron and the fibers it innervates are called a motor unit (MU). Muscle fibers of a MU are randomly distributed in the muscle (MU territory) and each axon reaches the fibers by the neuromuscular junctions. The pool of neuromuscular junctions of the fibers belonging to a $\mathrm{MU}$ is distributed in a territory, termed innervation zone (IZ).

The electric impulse that propagates along the motoneuron and reaches the neuromuscular junction determines the excitation of the muscle fiber membranes and the generation of propagating action potentials. A transmembrane current distribution (depolarization zone) corresponds to this potential distribution. The depolarization zones propagate without attenuation along the muscle fibers from the neuromuscular junctions to the two tendon endings (fig. 1). The velocity with which the action potential propagates depends on the fiber diameter and type and is termed muscle fiber conduction velocity $(\mathrm{CV})$. The intracellular action potentials generate and extinguish at the neuromuscular junctions and tendons, respectively. The summation of the action potentials generated by fibers innervated by a single motoneuron determines the MU action potential.

Each depolarization zone can be seen as a moving source of electric field at some depth below the skin. If the source moves along the fiber, the surface potential distribution will move with it. An electrode system placed on the skin will detect an interference signal due to the contributions of the action potential trains of all the active MUs. Increasing contraction force results in activation (recruitment) of an increasing number of progressively larger MUs and to an increase of the frequency of activation (firing rate) of those already active (Henneman's principle). The set of activation instants of a MU is termed firing pattern. 
a1
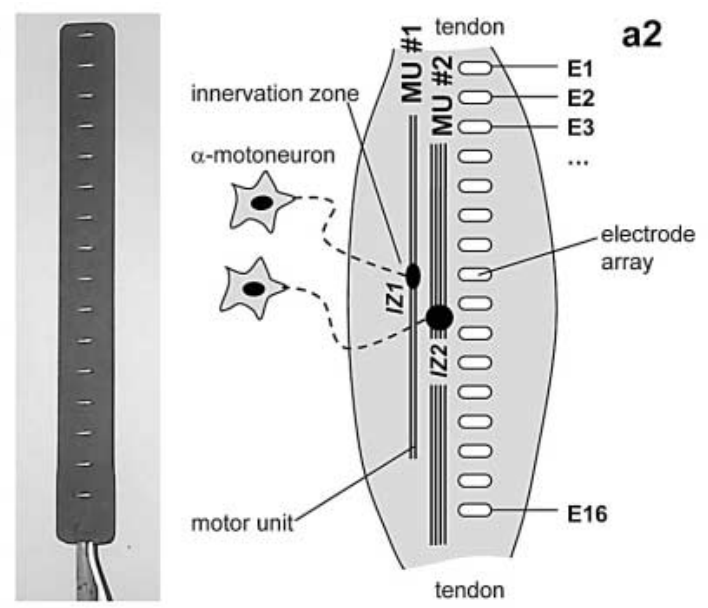

b1
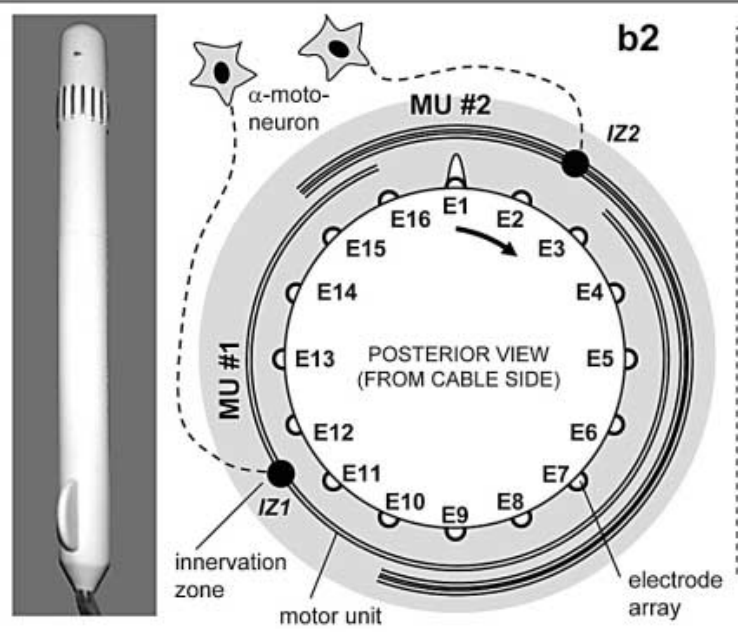

b2

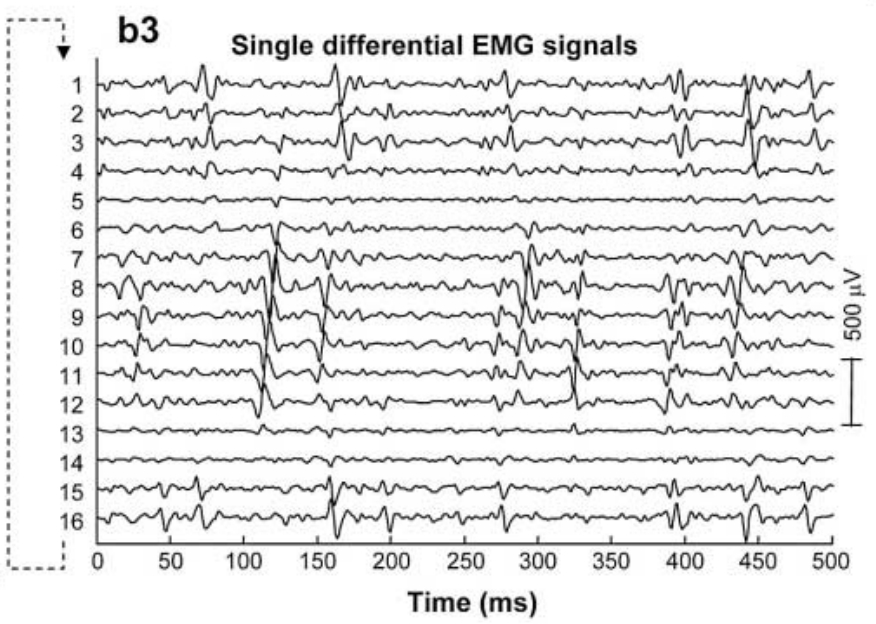

a3 Single differential EMG signals

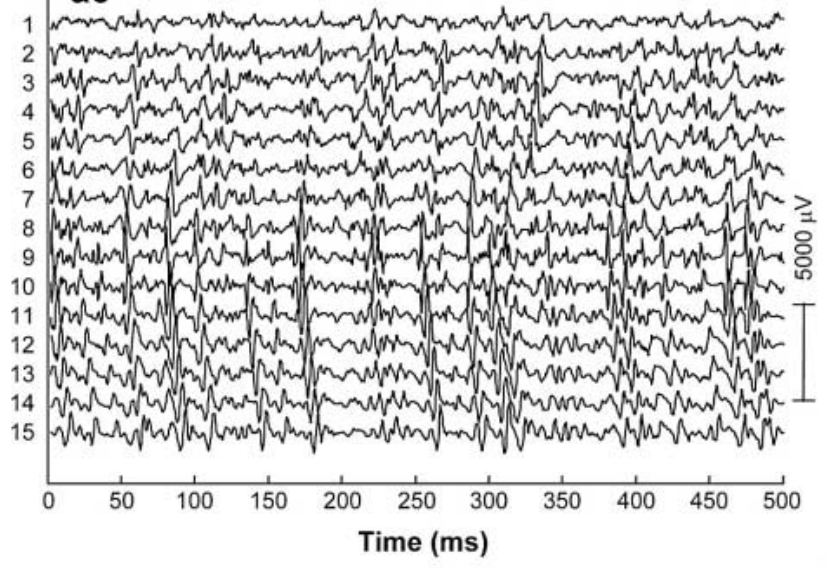

\section{b3}

Fig. 1. Principles of multichannel surface EMG detection with linear electrode arrays from skeletal muscles $(\mathbf{a 1}, \mathbf{a 2}, \mathbf{a 3})$, and with circular arrays from anal sphincter muscles (b1, b2, b3). a1 16-channel linear electrode array, formed by a flexible support holding 16 silver bar electrodes ( $5 \mathrm{~mm}$ length, $0.8 \mathrm{~mm}$ diameter), equally spaced by an inter-electrode distance (i.e.d.) of $10 \mathrm{~mm}$. a2 Schematic representation of the linear array location on a skeletal muscle (e.g. biceps brachii); two motor units (MU\#1 and MU\#2), characterized by different length, number of fibers and position of the innervation zone (IZ1 and IZ2) are depicted as an example. The array is aligned with fiber direction. a3 Sample epoch, $500 \mathrm{~ms}$ long, of multichannel single dif- ferential EMG signals detected with a 16-electrode, $10 \mathrm{~mm}$ i.e.d. array on a biceps brachii muscle at maximum contraction level. b1 16-channel anal probe. b2 Schematic representation of anal probe position with respect to the external anal sphincter muscle; two motor units (MU\#1 and MU\#2), characterized by different length, number of fibers and innervation zone positions (IZ1 and IZ2) are depicted as an example. b3 Sample epoch, $500 \mathrm{~ms}$ long, of multichannel single differential EMG signals detected with the probe shown in b1 from external anal sphincter muscle at maximum contraction level. Some MUs seem to be innervated at one extremity.
Figure 1a shows the characteristics of EMG signals detected at different locations along the biceps brachii muscle. The detection is performed by a number of equally spaced bipolar recordings, located along a line. This multichannel system is also known as linear electrode array [15-17]. The array detects signals with similar shape, which propagate in two opposite directions starting from the innervation zone. The basic idea is to locate electrodes along the muscle fiber orientation covering the entire muscle length.
Anatomy of the Anal Sphincter

The anatomy of the anal sphincter is rather complex. The EAS is a striated muscle whose fibers can be arranged circularly or can decussate anteriorly, towards the center of the perineum, differently for males and females $[18,19]$. The circumferential fibers can be divided into three annular sections, usually named subcutaneous, superficial and deep parts (going from the anal orifice towards the coccyx). The annular parts are traversed and joint by strands of longi- 
tudinal fibers. The deep part of the EAS closely blends with the puborectalis muscle and, usually, there is no clear separation between the two muscles.

\section{Design of an Array for the EAS}

Following the concepts behind the design of a linear array, in the case of the EAS it is necessary to locate electrodes around circumferences in order to follow the main muscle fiber orientation. As in the case of electrodes displaced longitudinally along rectilinear fibers (fig. 1a), the displacement of electrodes along a circumference allows the detection of the MU action potentials from their generation at the innervation zone to their extinction at the tendon endings. For this purpose a specific probe was designed [13,14].

The anal probe (fig. 1b1) is composed of a rounded-tip plastic cylinder, $150 \mathrm{~mm}$ in length and $14 \mathrm{~mm}$ in diameter, holding a circumferential array of 16 equally spaced silver bar electrodes, located at a distance of $20 \mathrm{~mm}$ from the probe tip and aligned with the probe axis. A flexible, multiwire cable encapsulated in silicone rubber is provided at the bottom of the probe, and is used to connect the circular array to a multichannel EMG amplifier.

A small plastic marking, encapsulated in the probe tip, indicates electrode 1 and the direction of numbering. A plastic fin at the end of the probe, also aligned with electrode 1 , helps the operator in checking the orientation of the probe with respect to a fixed reference during and after insertion.

The probe is manufactured using a purposefully designed machinery, which injects melted bio-compatible plastic (polystyrene) at a temperature of $250^{\circ} \mathrm{C}$ into a metallic mould, with a pressure of $120 \mathrm{~atm}$ and a variable injection speed. The probe can be sterilized chemically and is autoclavable.

By means of a multichannel electromyograph for surface EMG signals to which the probe is connected, each bipolar signal is amplified, band-pass filtered and acquired by a PC equipped with an analog-to-digital (A/D) conversion board and with a purposely designed acquisition and display software. Galvanic insulation through optical coupling is present between the probe and the acquisition system, providing a high degree of safety to the patient and protection from electrical shocks.

Figure 1b shows signals detected with the system described above. Similar signal features as in the case of other muscles (e.g. fig. 1a) can be recognized. In particular, the action potentials propagating from the innervation zone and terminating at the fiber endings can be detected from the multichannel recordings.

\section{Interpretation}

The interpretation of multichannel EMG signals recorded by the EAS probe (fig. 1b) requires advanced tools of signal processing and modelling. Two of them are briefly presented in the following.

\section{Decomposition}

The multichannel recordings are the summation of the action potentials of the active MUs. The decomposition of surface EMG signals is the procedure for the detection and extraction of the contributions of the single MUs. The possibility to track the activity of single MUs allows study of the central and peripheral properties of the neuromuscular system, such as motor control strategies and MU anatomical and physiological properties. For the purpose of decomposition, double differential signals (obtained by subtraction of two consecutive bipolar recordings) are often used to enhance the selectivity of the detection.
A software tool for the analysis of single MU properties developed by Gazzoni et al. [20] was applied to signals detected from the EAS using the probe described above. The method is automatic, without interaction with the operator, and involves a segmentation phase and a classification procedure (to detect action potentials and identify the MUs to which they belong) which adapts to slow changes of the MU action potential shapes.

Surface EMG signals detected with electrode arrays provide more information with respect to each signal considered independently. The partial redundancy (i.e. the observation of the same phenomena from different detection points) of the information provided by multichannel detection can be advantageously used by the decomposition technique for $\mathrm{MU}$ action potential identification, allowing the identification of discriminative information for the classification. At this moment, the method is not able to resolve superpositions of MU action potentials; for this reason, the detection of almost all the activation instants of the MUs significantly contributing to the signal is possible only in specific cases. In general, an incomplete firing pattern is extracted.

\section{Models}

A model is a set of equations describing a physical system which allows, to a certain extent, to predict the changes of the system as a consequence of modifications in the parameters. One of the greatest problems when studying a mathematical model of a physiological system is the biological complexity, which requires the introduction of important approximations in the model. It is of fundamental importance to tackle the difficulties with a gradual approach, testing the hypothesis, fitting simulations with experiments, critically analyzing the improvements in the prediction and interpretation capabilities of the models. Indeed, the detailed geometry and parameters are usually unknown, so that a precise model is usually not available. Furthermore, the parameters change among subjects, so that a set of parameters properly selected for a subject may be useless when studying another individual. Besides these limitations, modelling has an important role in the interpretation of experimental results since it provides (a) indications about the sensitivity of signal features to the physiological mechanisms under study, and (b) estimation of system parameters that cannot be measured directly. For example, starting from a simple mathematical model of a sphincter as a perfect cylindrical muscle [21,22], as shown in figure 2, numerical experiments can be performed to generate the surface EMG detected in different conditions, varying anatomical or physiological parameters, such as fiber length or position. Such simulations can be useful to compare detection system performance in different conditions, to improve their design or select their best way of use. Moreover, comparing experimental data with the simulations, it is possible to infer the value of unknown parameters or of the actual geometrical configuration.

\section{Results}

In the following we will present representative applications of the multichannel surface EMG detection system and processing techniques presented above. 


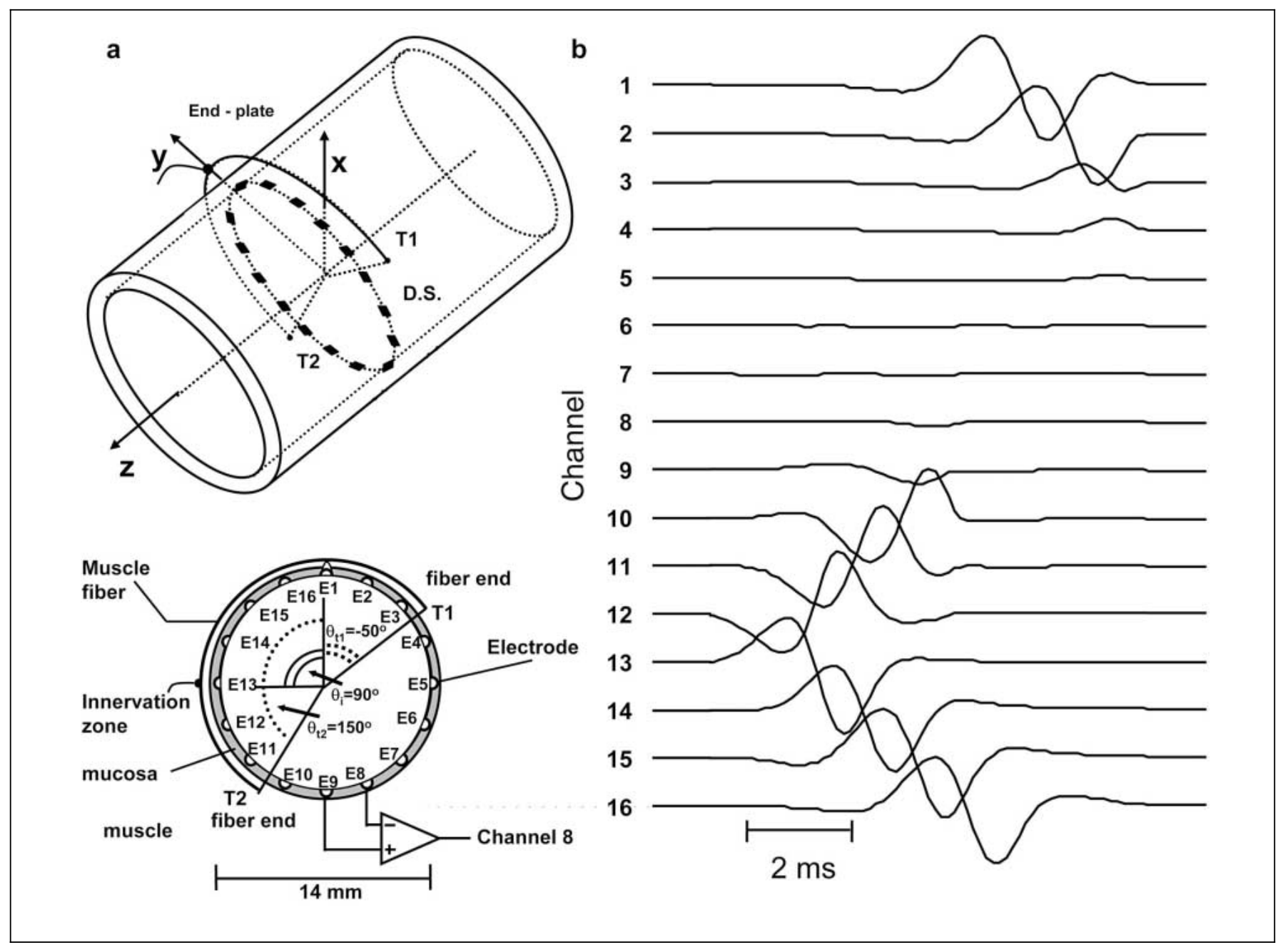

Fig. 2. a Geometry of a mathematical model of a sphincter as a circular cylinder [21]. b Example of simulation of single fiber differential surface EMG signals detected with 16 channels. The muscle fiber is $1 \mathrm{~mm}$ deep within the muscle. The mucosa is $1 \mathrm{~mm}$ thick. The innervation zone is at $+90^{\circ}$, the tendons at $+150^{\circ}$ and $-50^{\circ}\left(0^{\circ}\right.$ corresponding to the dorsal side and to electrode E1). The relative short duration of the simulated action potential is due to the generation of a single fiber potential, to the small fiber depth, and to the specific selection of geometry and conductivities of the anisotropic muscle tissue. Due to circular configuration, electrodes 16 and 1 are adjacent; as a consequence, the potential propagating from the channels over the IZ (channels 12 and 13) to channel 16 continues its propagation from channel 1 to channel 4. D.S.: Detection system.

\section{EMG Signal Amplitude}

Surface EMG amplitude may be indicative of the exerted force [23], thus it has been used for this purpose in many clinical studies. However, amplitude indicators (such as the average rectified or the root mean square value) are very sensitive to factors other than the relative degree of muscle activation. The detection of signals in many points over the muscle allows to analyze the sensitivity of signal features to electrode location. Figure 3 shows signals detected by the anal probe described above. The 16 signals obtained by the bipolar systems show sig- nificantly different amplitudes. As expected (and demonstrated elsewhere [3]), the signals detected in proximity of the innervation zones or tendon endings have a lower amplitude than the others. Different electrode configurations provide signals with different amplitude and spectral characteristics. As an example, figure 3 reports the signals detected by three bipolar systems with electrodes symmetrically placed. The figure also shows how the orientation of the electrode may affect the signal amplitude.

The variability of amplitude measurements is significantly reduced if the proper electrode location is selected 


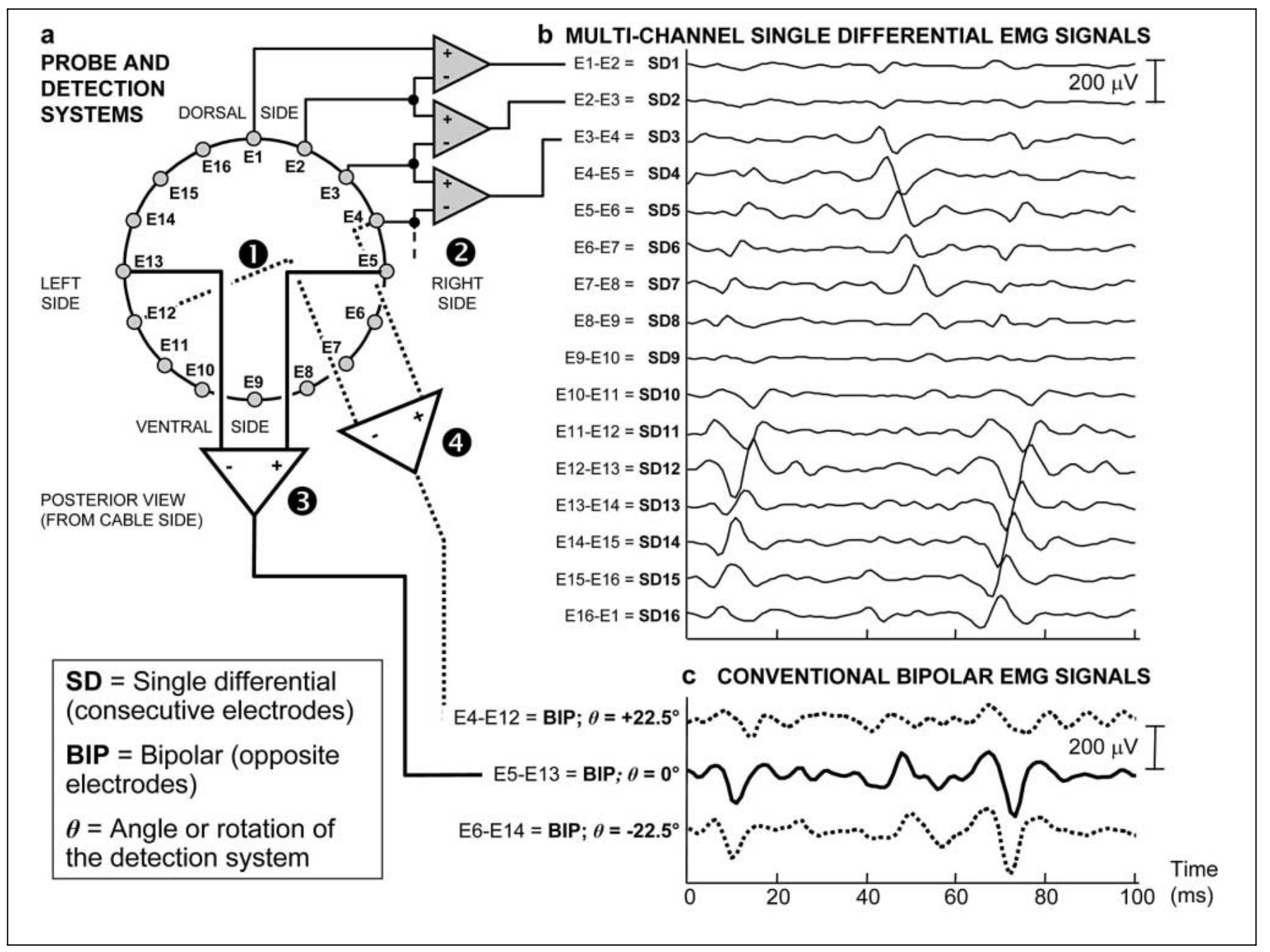

Fig. 3. Examples of surface EMG detection from the anal sphincter muscle using different detection systems. a Schematic representation of the rectal probe (1), shown from cable side (see also fig. $1 \mathrm{~b} 1$ and corresponding caption). Multichannel EMG detection is obtained with a series of differential amplifiers (2). Conventional bipolar EMG recording is instead obtained detecting a single signal as the difference between two opposite electrodes (3), from which only amplitude-based information can be extracted. (4) Effect of rotation of a bipolar probe by a one-electrode step $\left(22.5^{\circ}\right)$ in counter-clockwise direction. b Sample epoch, $100 \mathrm{~ms}$ long, of multichannel single differential EMG signals detected from external anal sphincter mus- cle during maximal contraction, using the probe shown in figure $1 \mathrm{~b} 1$, and the detection method schematized in (2). c Sample epoch of conventional bipolar (BIP) EMG signals, calculated from opposite electrodes on the same signal shown in $\mathbf{b}$, in three different probe orientations: electrodes aligned to left-right direction (BIP; $\theta=0^{\circ}$, solid line), rotated by $22.5^{\circ}$ counter-clockwise (BIP; $\theta=+22.5^{\circ}$ ), and clockwise (BIP; $\left.\theta=-22.5^{\circ}\right)$. In this specific case, a slight $\left(\theta=-22.5^{\circ}\right)$ rotation of the probe in clockwise direction would not produce a significant effect, while a rotation in the other direction would greatly affect both the amplitude (by about a twofold factor) and shape of the potentials. specifically in each recording condition. A possible criterion is to estimate signal amplitude from bipolar arrangements located between the innervation zone and the tendon endings. In this case, the maximum amplitude is obtained. A multichannel measurement is required to identify the optimal position.

Multichannel EMG from the Anal Sphincter

\section{Non-Invasive Assessment of Muscle Anatomical}

\section{Properties}

Multichannel EMG signals allow to obtain important information about anatomical properties of the muscle under study [3, 15-17, 24-26]. According to the concepts described above (see also fig. 1), visual analysis of the 
Fig. 4. Examples of multichannel EMG signals detected from the external anal sphincter muscle of a female subject. Signals were acquired from $1 \mathrm{~cm}$ depth in the anal canal. a Relaxed condition. b Maximal voluntary contraction. Note the different vertical scales in $\mathbf{a}$ and $\mathbf{b}$.

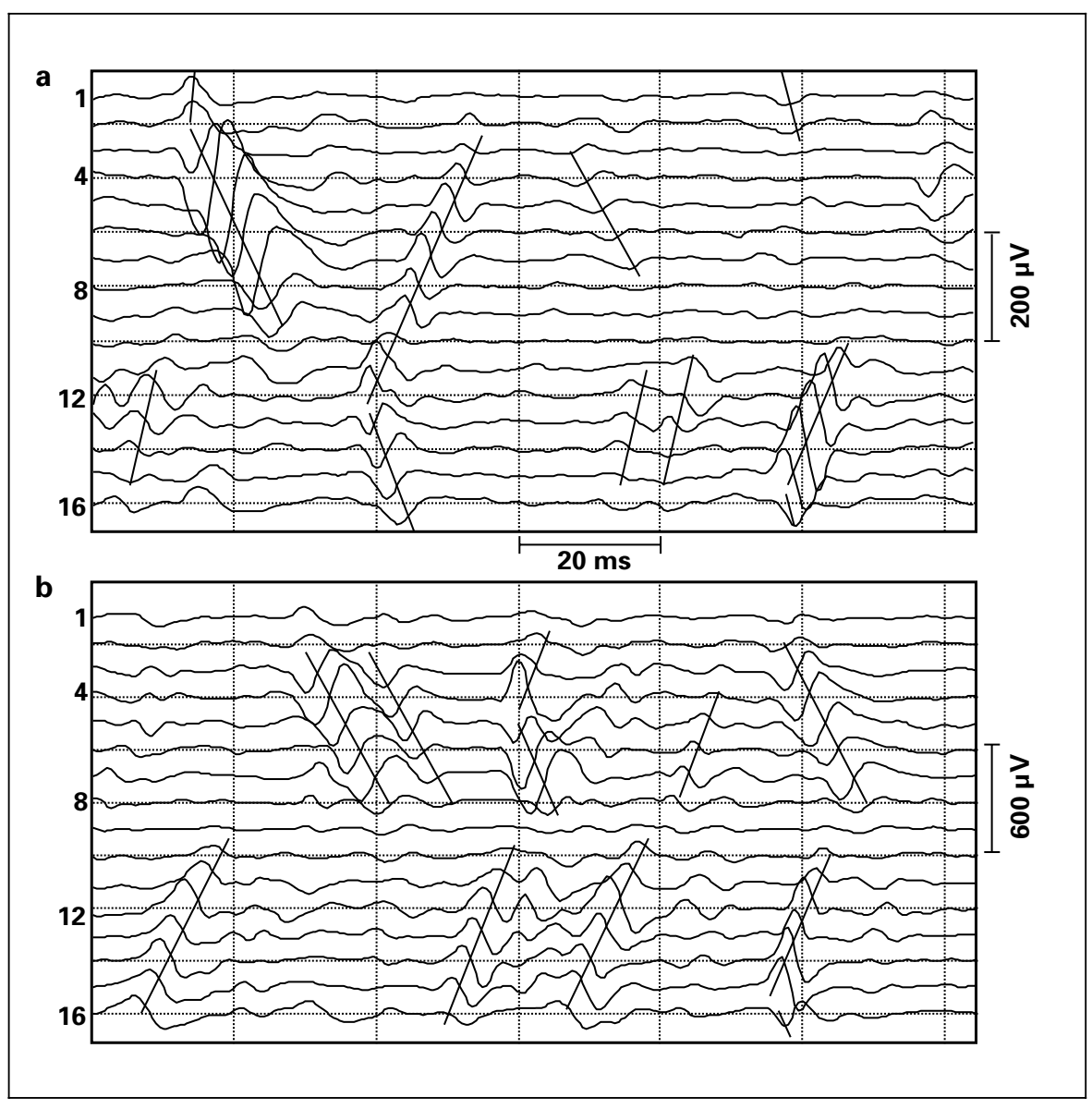

multichannel recordings allows estimation of the length of the muscle fibers, the location of the innervation zones and of the tendon regions. Figure 4 shows signals detected from the EAS with the visual identification of the MU anatomical features (innervation zones and fiber length). The MUs are innervated at different locations. The potentials propagate from the innervation zone, towards the fiber endings, with a specific $\mathrm{CV}$.

\section{Detection of Single Motor Unit Activities}

The application of the decomposition technique described above to signals acquired from the sphincter muscle showed that it is possible to non-invasively identify MUs at low and high contraction levels. In many cases, it was possible to detect the same MUs at different contraction levels as well as the progression and the recruitment of new ones with increasing effort.

Figure 5 shows an example of decomposition of two signals recorded at maximal and intermediate contraction levels from a pathological subject. At maximal contraction level four MUs are detected. By decreasing the contraction intensity, two MUs are de-recruited. The same two MUs are active at both contraction levels. Firing pattern of MU 2 is rather well reconstructed for both contraction levels. Since the symptomatic subject was not able to maintain the intermediate contraction, the activity gradually decreased from $t=2 \mathrm{~s}$ and stopped at $t=7 \mathrm{~s}$. This behavior is well described in the de-recruitment pattern shown in the right column diagrams of figure 5.

\section{Estimation of Muscle Fiber Conduction Velocity}

Muscle fiber $\mathrm{CV}$ is an important physiological parameter since it reflects muscle fiber type and contractile properties [27]. Conduction velocity can be estimated from multichannel surface EMG signals by computing the delay of propagation between signals detected by systems placed along the fiber direction [28]. In case of sphincter muscles, the specific geometry of the muscle makes the 


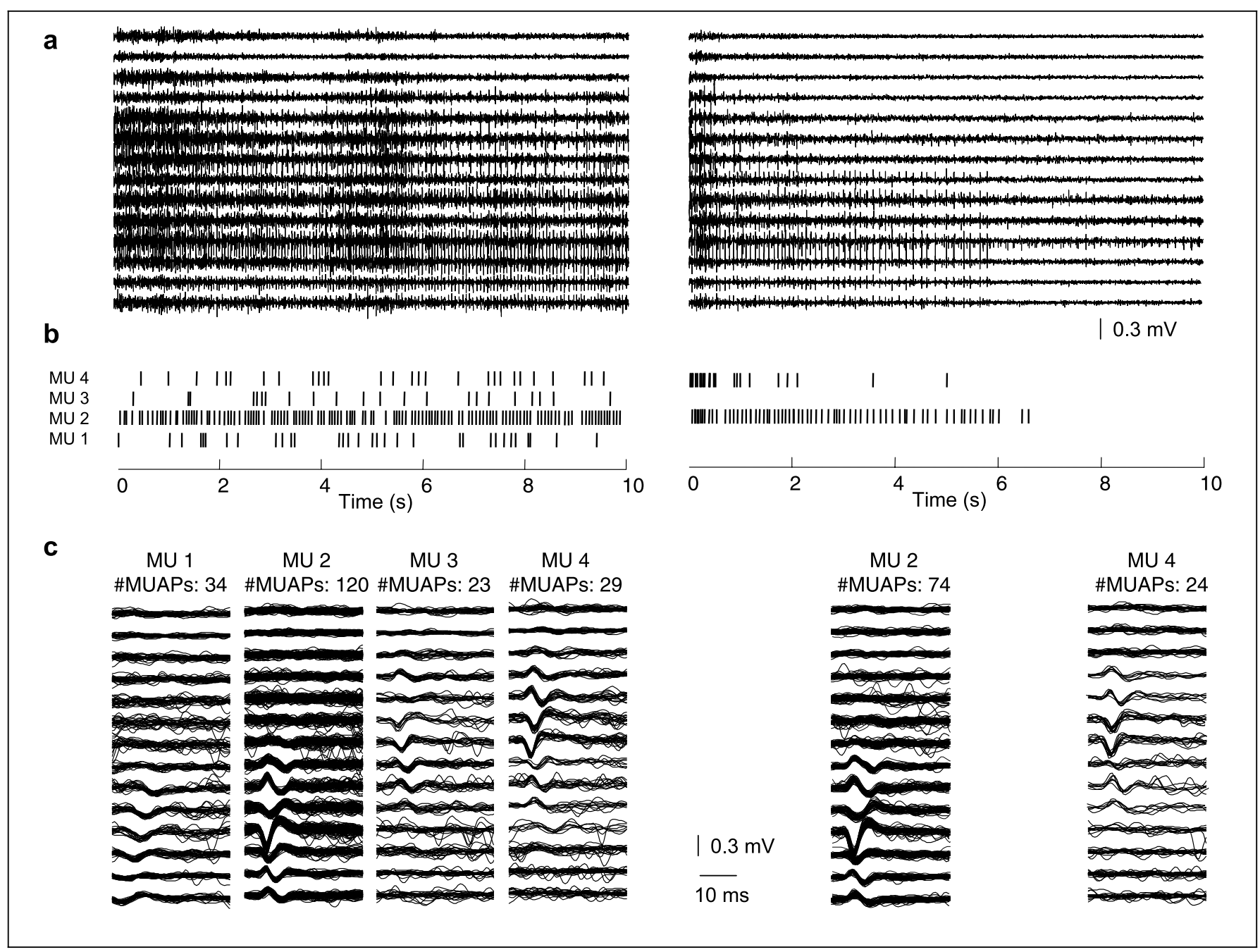

Fig. 5. Example of the decomposition of signals recorded during $10 \mathrm{~s}$ long contractions at the maximal (on the left) and intermediate (on the right) contraction levels. a Raw signals. b Firing patterns of the identified MUs. c Superposition of the MU action potentials belonging to each of the four MUs. Note that the same MUs (\#2 and \#4) are identified at the two contraction levels and new MUs (\#1 and \#3) are recruited at the maximal effort. In particular, MU\#2 firing pattern is quite well reconstructed.

estimation of CV critical. Indeed, the observed delay of propagation depends not only on the velocity of propagation but also on the location of the muscle fibers within the muscle (fig. 6). Methods for estimating CV from these muscles should be based on the concomitant estimation of the source depth and of the propagation delay. Results from limb muscles indicate that this goal is feasible from two-dimensional surface EMG recordings [29, 30].

\section{Discussion and Conclusions}

In this work an innovative technique for the detection of surface EMG signals from the anal sphincter muscles was presented. The multichannel method allows getting additional information with respect to classic bipolar recordings. The use of an internal plug, as required in the technique herein presented, does not significantly alter the muscle functions and the contractile mechanisms [7].

When single electrode bar pairs or pads are adopted, their position with respect to the anatomical characteris- 


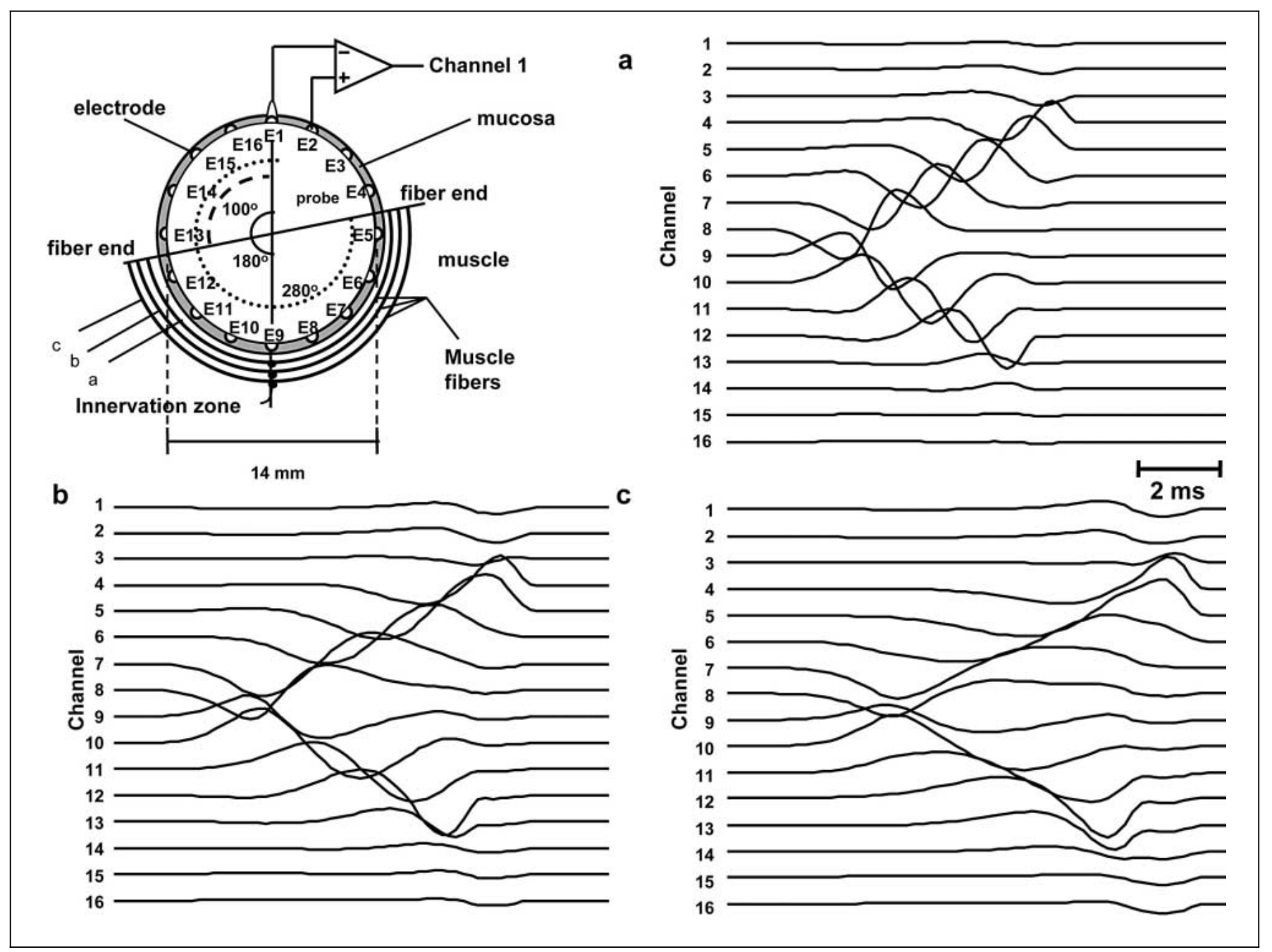

Fig. 6. Simulation of single differential EMG signals generated by a single fiber at three different depths within the muscle. The model is a two-layer circular cylinder [21] with muscle and mucosa ( $1 \mathrm{~mm}$ thick). The innervation zone is at $180^{\circ}$, the fiber ends at $100^{\circ}$ and $280^{\circ}\left(0^{\circ}\right.$ corresponding to the dorsal side and to electrode E1). The fibers are $1,2.5$ and $4 \mathrm{~mm}$ deep within the muscle. The set of signals reported in $\mathbf{a}, \mathbf{b}$ and $\mathbf{c}$ are normalized with respect to the maximum amplitude of each.

tics of the sphincter muscles is of pivotal importance [7]. Binnie et al. [7], comparing the use of two rings placed transversally in the sphincter canal with respect to the use of two flat bars placed longitudinally, concluded that the latter was better than the former since higher signal amplitude was recordable with that arrangement. The array technique herein adopted and described in detail elsewhere $[15,16,25,31]$ allows to interpret those findings. Since MU action potentials propagated along the fibers placed circularly around the sphincter canal, the information provided by the ring arrangement corresponded to the variation of EMG activity with respect to the depth in the canal. On the contrary, since the two bars placed along the canal length averaged the differences in that longitudinal direction, they provided the EMG amplitude differences, along the circumference between the two sides, strongly affected by the low-pass filter due to the averaging effect along the canal length. EMG amplitude differences between the two opposite canal sides are one order of magnitude greater than differences between two different depths within the canal (which is in the range of tens of microvolts, comparable to the noise of the skin-electrode contact, and is thus difficult to appreciate). 
The signals presented in this study (fig. 1) carry information on muscle anatomy, about the localization of the innervation zone(s), the length of the fibers, the degree of symmetry between the two sides of the anal muscle. EMG signals are recorded from a small muscle portion since electrode dimensions are smaller than those traditionally adopted. In such a way, crosstalk (i.e. the detection of electrical activity from neighboring muscles) and low-pass smoothing effects are diminished [32, 33]. Moreover, the decomposition technique allows to identify single MU action potentials and, in particular conditions, to track their activity during contraction at different force levels (fig. 5).

This information, extracted noninvasively, is promising for future understanding, care and monitoring of the pathologies related to pelvic floor disorders. They provide a number of quantitative variables and parameters currently not available with other approaches. The use, for instance, of adhesive electrodes directly placed externally to the sphincter, allows to record EMG signals, but, as clearly depicted in the figures reported in the study by O'Donnell et al. [8], the electrode dimensions, their contact surface and their location on the external sphincter generate signals affected by too many factors which reduce the reproducibility of the test.

In conclusion, the detection of many surface EMG signals, along the fiber direction, from the EAS provides additional information with respect to previously proposed techniques. This opens interesting perspectives for diagnostic and monitoring applications in the pelvic floor.

\section{Acknowledgements}

This work was supported by the European Shared Cost Project On ASymmetry In Sphincters (OASIS) (Contract No. QLK6-CT2001-00218), the Regional Health Administration of Piemonte, Fondazione Cassa di Risparmio di Torino, and Compagnia di San Paolo, Torino, Italy.

\section{References}

1 Farina D, Merletti R, Enoka RM: The extraction of neural strategies from the surface EMG. J Appl Physiol 2004;96:1486-1495.

2 Jensen $\mathrm{C}$, Vasseljen $\mathrm{O}$, Westgaard RH: The influence of electrode position on bipolar surface electromyogram recordings of the upper trapezius muscle. Eur J Appl Physiol Occup Physiol 1993;67:266-273.

3 Roy SH, De Luca CJ, Schneider J: Effects of electrode location on myoelectric conduction velocity and median frequency estimates. J Appl Physiol 1986;61:1510-1517.

4 Hermens HJ, Freriks B: The state of the art on sensors and sensor placement procedures for surface electromyography: A proposal for sensor placement procedures. Deliverable of the SENIAM Project 1997; Roessingh Research and Development (Pub.)

5 Haig AJ, Gelblum JB, Rechtien JJ, Gitter AJ: Technology assessment: The use of surface EMG in the diagnosis and treatment of nerve and muscle disorders. Muscle Nerve 1996;19: 392-395.

6 Pullman SL, Goodin DS, Marquinez AI, Tabbal S, Rubin M: Clinical utility of surface EMG: report of the therapeutics and technology assessment subcommittee of the American Academy of Neurology. Neurology 2000;25;55: 171-177.

7 Binnie NR, Kawimbe BM, Papachrysostomou M, Clare N, Smith AN: The importance of the orientation of the electrode plates in recording the external anal sphincter EMG by non-invasive anal plug electrodes. Int $\mathbf{J}$ Colorectal Dis 1991;6:5-8.
8 O’Donnell P, Beck C, Doyle R, Eubanks C: Surface electrodes in perineal electromyography. Urology 1988;32:375-379.

9 Nielsen KK, Kristensen ES, Qvist N, Jensen KM, Dalsgard J, Krarup T, Pedersen D: A comparative study of various electrodes in electromyography of the striated urethral and anal sphincter in children. Br J Urol 1985;57:557559.

10 Kiesswetter H: EMG-patterns of pelvic floor muscles with surface electrodes. Urol Int 1976; 31:60-69.

11 Farina D, Cescon C, Merletti R: Influence of anatomical, physical, and detection-system parameters on surface EMG. Biol Cybern 2002; 86:445-456.

12 Zwarts MJ, Stegeman DF: Multichannel surface EMG: basic aspects and clinical utility. Muscle Nerve 2003;28:1-17.

13 Merletti R, Enck P, Gazzoni M, Hinninghofen $\mathrm{H}$ : Surface EMG recording of single motor unit action potentials from the external anal sphincter. Proceedings of the XIV ISEK Congress, Vienna, 2002, pp 23-24.

14 Enck P, Franz H, Azpiroz F, Fernandez-Fraga $\mathrm{X}$, Hinninghofen H, Kaske-Bretag K, Bottin A, Martina S, Merletti R: Innervation zones of the external anal sphincter in healthy male and female subjects: Preliminary results. Digestion 2004;69:123-130.
15 Merletti R, Farina D, Granata A: Non-invasive assessment of motor unit properties with linear electrode arrays. Electroencephalogr Clin Neurophysiol Suppl 1999;50:293-300.

16 Merletti R, Farina D, Gazzoni M: The linear electrode array: A useful tool with many applications. J Electromyogr Kinesiol 2003;13:3747.

17 Masuda T, Miyano H, Sadoyama T: The position of innervation zones in the biceps brachii investigated by surface electromyography. IEEE Trans Biomed Eng 1985;32:36-42.

18 Oh C, Kark A: Anatomy of the external anal sphincter. Br J Surg 1972;59:717-723.

19 Schuster M, Crowell M, Koch K: Atlas of Gastrointestinal Motility in Health and Disease. New York, Decker, 2002.

20 Gazzoni M, Farina D, Merletti R: A new method for the extraction and classification of single motor unit action potentials from surface EMG signals. J Neurosci Methods; in press.

21 Farina D, Mesin L, Martina S, Merletti R: A surface EMG generation model with multilayer cylindrical description of the volume conductor. IEEE Trans Biomed Eng 2004;51:415426.

22 Farina D, Mesin L, Martina S: Advances in surface electromyographic signal simulation with analytical and numerical descriptions of the volume conductor. Med Biol Eng Comput; in press.

23 Bigland-Ritchie B: EMG/force relations and fatigue of human voluntary contractions. Exerc Sport Sci Rev 1981;9:75-117. 
24 Masuda T, Miyano H, Sadoyama T: The distribution of myoneural junctions in the biceps brachii investigated by surface electromyography. Electroenceph Clin Neurophysiol 1983; 56:597-603.

25 Roeleveld K, Stegeman DF: What do we learn from motor unit action potentials in surface electromyography? Muscle Nerve 2002;11: S92-S97.

26 Rainoldi A, Melchiorri G, Lucchetti D, Caruso I: A method for positioning electrodes during surface EMG recordings in lower limb muscles. J Neurosci Methods 2004;134:37-43.

27 Andreassen S, Arendt-Nielsen L: Muscle fibre conduction velocity in motor units of the human anterior tibial muscle: A new size principle parameter. J Physiol 1987;391:561-571.
28 Farina D, Merletti R: Methods for estimating muscle fiber conduction velocity from surface electromyographic signals. Med Biol Eng Comput; in press.

29 Roeleveld K, Stegeman DF, Vingerhoets HM, Van Oosterom A: The motor unit potential distribution over the skin surface and its use in estimating the motor unit location. Acta Physiol Scand 1997; 161:465-472.

30 Roeleveld K, Sandberg A, Stalberg EV, Stegeman DF: Motor unit size estimation of enlarged motor units with surface electromyography. Muscle Nerve 1998;21:878-886.
31 Merletti R, Rainoldi A, Farina D: Surface electromyography for noninvasive characterization of muscle. Exerc Sport Sci Rev 2001;29: 20-25.

32 Farina D, Rainoldi A: Compensation of the effect of sub-cutaneous tissue layers on surface EMG: A simulation study. (Special Issue on Intelligent data in electromyography and electroneurography.) Med Eng Phys 1991;21:487496.

33 Farina D, Merletti R, Indino B, Nazzaro M, Pozzo M: Surface EMG crosstalk between knee extensor muscles: Experimental and model results. Muscle Nerve 2002;26:681-695. 